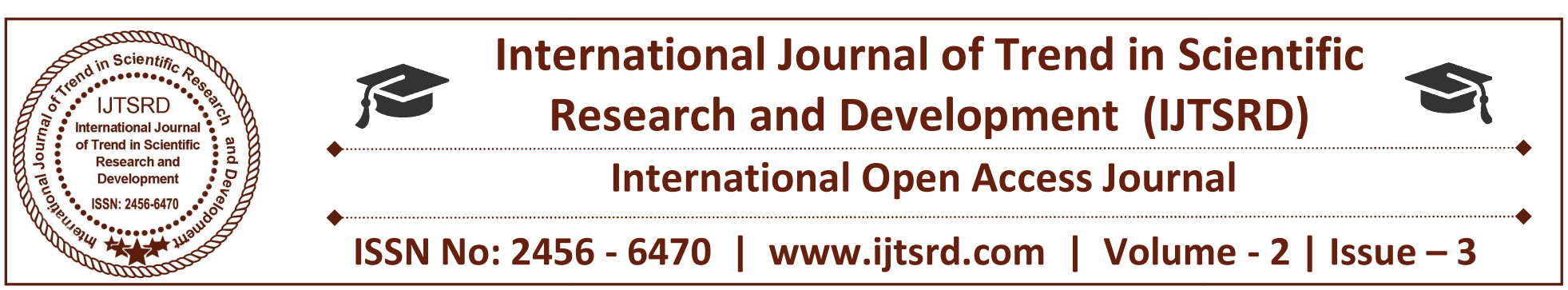

\title{
A Secure Multi Agent VANET Model to Improve Communication Services in VANET
}

Vivek Shrivastava

M.Tech Scholar, Department of Computer Science \& Engineering, Sagar Institute of Research \& Technology, Bhopal, India

\author{
Sunil Malviya \\ Assistant Professor, Department of Computer \\ Science \& Engineering, Sagar Institute of \\ Research \& Technology, Bhopal, India
}

\section{ABSTRACT}

In the recent decade the VANET has been emerged as a prominent field for research and it receives considerable attention. VANET uses different specifications of the WLAN 802.11 family. It takes the basics of ad hoc network and the VANET creates a network with a collection of independent entities with the ability to communicate among them. The Adaption to the various 802.1 family causes a remarkable increase in the number of wireless network. The VANET has various benefits along with many challenges including security, privacy, Quality of communication, effective bandwidth utilization etc. The importance of Wireless Distributed network in current scenario is very major in communication technology is does through multiple cooperating clients. We know network users are growing in day to day life it does heavy load over the network and the traffic patterns are also getting dynamic, it does affect networking performance and quality in services, it require to get split out the network load into multiple server or client areas, it also does challenges for Wireless Distributed applications, in this case we need to develop such type of Wireless Distributed network that can create peer to peer networking in cycle, need to integrate network and application so that servers and clients can handle without networking complexity. In this thesis we propose an Agent Based Routing Scheme through which support routing with high number of nodes without flooding so that network bandwidth can be maximum utilizes. Proposed model provides a new Reactive routing algorithm that perform higher throughput and lower delay compare to the traditional VANET protocol like AODV and DSR.

Keywords: VANET, Agent based Routing Scheme, AODV, DSR, WLAN 802.1

\section{INTRODUCTION}

VANET has led to the ubiquity of wireless networks for personal, commercial, industrial, and military use. In many applications, device location plays a critical role in network operations and services. Advances in distributed systems with increasingly growing capabilities for efficient file transport and their immediate Consequence, i.e. the ability to rapidly replicate content over a network, has made sharing of electronic files become a revolution in business and domestic environments. The high level of decentralization, dynamism network management, and self-management of a number of emerging communication environments, including teamwork, pure wireless distributed network and mobile ad hoc networks $(\mathrm{ABC})$, in this way no of clients are participated in the process of communication or collaboration without relying on central authorities, enforce cooperation to play an essential role in the overall network functioning. Particularly, ad hoc networks rely upon the cooperation among individual nodes to carry out essential tasks such as packet forwarding. VANET network file sharing systems face a similar situation. File sharing has become a common practice for Internet users to obtain, for example, software updates from public sites. 
However, such a practice still provokes mistrust. File corruption may occur easily through dishonest and malicious actions or even by mistake. Similarly, an impostor could masquerade himself as the originator of a certain file, publishing a corrupted version of the file. In fact, users of currently deployed file sharing systems are unable to verify that files they retrieve are uncorrupted, or whether the content has been truly created by the presumed owner. Providing higher level of security in VANET or ad hoc networks is an active research area which prompts many challenges. Most of the business are doing through the networking devices and technology such storage devices are good for digitalization but it comes hard when volume of request and response gets higher due to heavy traffic load, users producing heavy traffic , which need to be manage by network technology, Now are days internetworking is growing across the country it does affect quality of services factors that put stress over the centralized server of network in this duration data loss, frame delay and lower throughput has been monitor by the system in this case so many time user having reliability issues degrades the quality of data communication, all the intermediate devices is not performing good due to heavy traffic during transmission such problem issues motivates so many researchers from long time to resolve and maintain traffic peer to peer network error controls so many papers and technology has been proposed for solution but the previous technology and protocol is not sufficient for this purpose, therefore we need to create and develop such type of mechanism that not only improve quality of service factors but also resolve data loss issues so that reliability and accountability can be achieve at some extent.

In VANET network an essential issue is the reliable routing policy. In wireless distributed network an essential issue is the reliable routing policy The $\mathrm{ABC}$ protocol is based on four respects, i.e.-resources distance, resources angle, the energy levels of resources and bandwidth link. The ABC protocol can improve the reliability and stability of the existing routing mechanisms with less energy consumption.

\section{RELATED WORK}

There are various techniques and algorithm has been proposed for VANET but still various challenges has been facing, many researchers are doing their work in this area

\section{A. Ad-Hoc On-Demand distance vector in VANET}

AODV routing protocol supports various routing as well as supportive communication services like the multi-hop routing among multiple mobile nodes for securing establishing and upgrading an mobile ad hoc network. AODV working is based upon the Destination-Sequence Distance Vector strategy worked as an algorithm. The difference from other ad hoc protocol is that AODV is reactive category of protocols, on the other hand DSDV is belongs to proactive. AODV works in the way in which it requests a route only when it is required, and it does not need any mobile nodes to gets routes to the destination.

To send or get connect a message to a destination node, a source node first initiates a route discovery investigation process to locate find the destination. A Route Request process control packet is works for flooded through the network activity until it gets to accurate destination or it reaches to a required node that having the details about routing to the destination node. On its way it will through the network nodes, the Resource REQ data unit it initiates the temporary routing information for the detail path at all the mobile nodes it passes.

On the other proceeding stage, Route Reply control packet will be used to find destination by uni-cast routing from source end it brings along the routing information temporary reverse route. Routes has been chosen by the route technique used for discovery process are works as follows. If a is suffered during the process of routing and if we found that route is divided due to the movement of the source mobile node, then it will it reprocess the route discovery procedure to get a new route to reach towards to its destination. If a route is divided since the movement of a mobile node in their route, its neighboring upstream mobile node notices such type of movement then it taken action and produces link failure message through its all remaining mobile nodes of the active upstream nodes to the source nodes routing of the next hope to gets its requested destination place.

\section{B. Destination Source Routing in VANET}

The Dynamic Source Routing protocol having many multiple verities like is a simple and robust routing protocol developed for support and use in wireless adhoc networks routing services of mobile nodes. The 
use of source mobile node routing allows data packets routing to be loop-free, in such a way it avoids need for up-to-date routing information at the intermediate mobile nodes via packets are to be transmitted, and it allows source nodes transmitting or to caching the routing information kept securely in them. Dynamic Source Routing is comes into the category of beaconless protocol.

During routing phase, it designs a RREQ which is flooded in entire network. The similar process used by destination nodes responds by having RREP, which carries the information in route by the RREQ packet. Each RREQ packet having a sequence number created by source node which is used to secure loop formation and to protect by multiple transmission of the same RREQ packets by intermediate mobile node that receives it through information paths. DSR is also belongs to a purely on-demand ad hoc network routing protocol like AODV. This means that a route is designed and invented in dynamic manner means only when it is needed and no preprocess of connectivity is performed during the routing process.

\section{LITERATURE SURVEY}

In [4] proposed a impressive novel approach named Performance Analysis of Wireless Distributed with Difference performance Control Mechanism, it is special designed for Internet, internet is growing technology at today's environment, so that traffic control is one of the issue of discussion, that motivates the researchers to resolve the problem with performance modeling, the proposed scheme presents a stochastic queuing model to control congestion and improve performance, scheme consist a new analytical model that works integrated with the collaboration of tradition RED model, model works on the basic principal of reduction of traffic load when channels get long delay, where packet has been going drop as per the need of the network scenario , algorithm perform evaluation of queue memory optimization, the system describes the performance valuation from different point of view like mean system occupancy and delay, evaluation of data loss and throughput has been considered by the system as a packet dropping model .

An efficient routing is an important factor for all WSN protocol in order to meet the node failure, intruder attacks and resource constraints. In the present study, a method is proposed for a hybrid secure routing protocol which offering a high level scalability, security, cluster formation and cluster head selection, so that the network life time can be increased gradually and the results are compared with the LEACH protocol. From the results, the proposed routing protocol can provide better quality of service, increased network life time, energy efficient and more secure routing for wireless sensor network than LEACH protocol.

According to [9] proposed a new research regarding the QoS control in area of multicasting communication over distributed Wireless Distributed network, their network should consist the policy of many to many relationship, such type of relationship is cooperate multimedia applications, to get stream delivery services, proposed research gets the time of -A friendly Active Layered Multicast quality Control Mechanismll such mechanism is effective and works with the help of an model called ALMCC , that uses active layer labeling and network adaptability, it specify that how the network state is growing as the network traffic going on and the throughput variation scenario to reflect the threshold measurement by different network state.

In [8] Mobile ad hoc networks (MANET) represent complex distributed systems that comprise wireless mobile nodes that can freely and dynamically self organize into arbitrary and temporary ad hoc network topologies. A mobile ad hoc network is a collection of nodes that is connected through a wireless medium forming rapidly changing topologies. The widely accepted existing routing protocols designed to accommodate the needs of such self-organized networks do not address possible threats aiming at the disruption of the protocol itself.

\section{PROPOSED MODULE}

In order to Design and Develop most Efficient Agent Based Communication Model for VANETs we will go through following proposed methodology:

- Module 1: Coverage and position based selfadaptive quick node deployment algorithm, in this algorithm we develop a new prototype/algorithm to make, place, move the nodes in the VANET network area.

- Module 2: We develop a agent based algorithm called Best resource picker reliable routing (BRPR) which should take the minimum hop count, no link failure, fast transfer, high 
throughput and packet delivery ratio with less energy consumption.

- Module 3: Pre-calculation step includes the process of node traverse direction, coverage area, energy level, linkage between nodes till the end of the simulation. Based on the pre-calculation result, we do the bandwidth adjustment algorithm to avoid the link failure.

- Module 4: Bandwidth adjustment algorithm will increase the bandwidth of the link which are in active and remaining node links bandwidth will get decrease since it is not participating in the transmission. When it conforms it is strong path, Start sending the packets do the same steps till the end of the simulation since it is ad hoc network.

\section{PROPOSED ALGORITHM AND FLOW CHART}

ABC (Agent Based Communication) algorithm has been implemented in following way to get best node picker to perform efficient communication at real time VANETs.

\section{Find the source node and destination node:}

- Find how many source and destination nodes are in the network.

- Based on number of source and destination, we need to provide the load balancing for the routing process.

- Send Request from destination to source with minimum number of hop by looking the Routing Table.

- Once source, got the request from the destination, the below process will get started.

- Make the empty routing table.

- Find the neighbor nodes list from the source node.

- Select the particular node which is available in all the 4 tables with high priority.

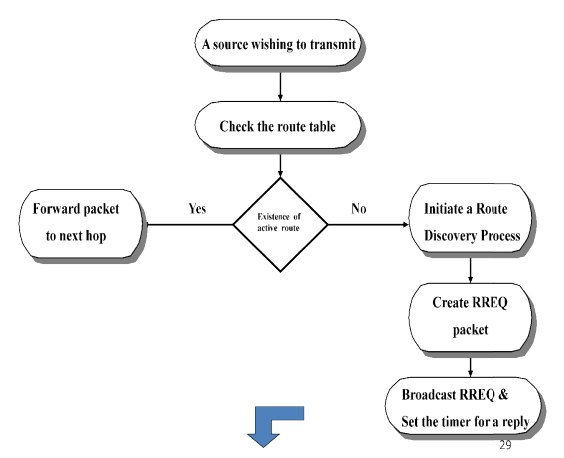

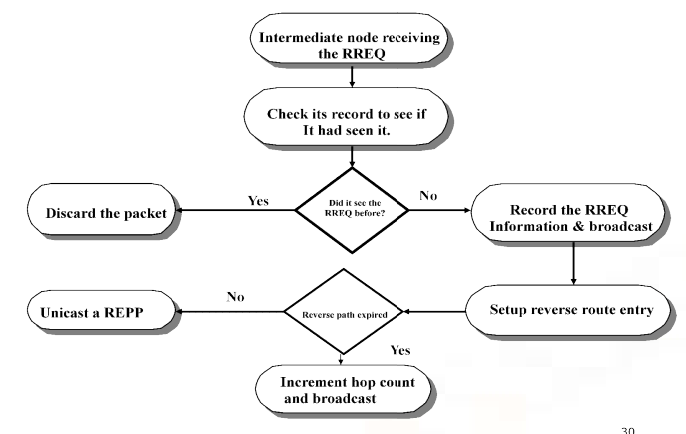

\section{Fig.1: VANET ABC Model for Performance Efficiency}

\section{CONCLUSION}

The main objective of this paper is to design a new Agent based Routing protocol for Vehicular Ad-hoc Networks (VANET). The challenges and limitations of the existing protocols in vehicular environments must be explored first in order to achieve this objective. Secondly, issues towards the design of the new $A B C$ protocol must be identified by using theoretical foundations and algorithmic methodologies.

\section{REFERENCES}

1. Nikita Lyamin, Alexey Vinel, Magnus Jonsson, And Boris Bellalta," Cooperative Awareness In Vanets: On Etsi En 302 637-2 Performance", Ieee Transactions On Vehicular Technology, Vol. 67, No. 1, January 201817.

2. Mahmoud Hashem Eiza," Situation-Aware Qos Routing Algorithm For Vehicular Ad Hoc Networks"Ieee Transactions On Vehicular Technology, Vol. 64, No. 12, December 2015.

3. Chang Guo, Demin Li,"Data Delivery Delay Reduction For Vanets On Bi-Directional Roadway", Received November 13, 2016, Accepted November 30, 2016, Date Of Publication December 15, 2016, Digital Object Identifier 10.1109/Access.2016.2640193.

4. Reza Shahidi,'On The Analytical Calculation Of The Probability Distribution Of End-To-End Delay In A Two-Way Highway Vanet", Received October 9, 2017, Accepted November 16, 2017, Date Of Publication November 27, 2017, Digital Object Identifier10.1109/Access.2017.2777963.

5. Manjot Kaur, Sukhman Kaur," VEHICULAR AD HOC NETWORKS" Volume 3, No. 3, March 
2012 Journal of Global Research in Computer Science TECHNICAL.

6. U.S. Department of Transportation, "Intelligent Transportation Systems (ITS) Home", http://www.its.dot.gov/index.htm, February 2012.

7. A.K. Saha and D.B. Johnson. "Modeling Mobility for Vehicular Ad Hoc Networks", Department of Computer Science, Rice University, Houston, USA.

8. Car-to-Car Communication Consortium, http://www.car-to-car.org., February 2012.

9. Z. Li, Z. Wang and C. Chigan, "Security of Vehicular Ad Hoc Networks for Intelligent Transportation Systems in Wireless Technologies", Nova Science Publishers, 2009. 